\title{
Effect of Advanced Strengthening Program on Physical Fitness Test Performance in Injury Prone Pre-Military Trainee Cadets
}

\author{
Mukul Khutale ${ }^{1}$, Sandeep Babasaheb Shinde ${ }^{2}$ \\ 1Department of Physiotherapy, Krishna Institute of Medical Sciences (Deemed to Be \\ University) Karad, Maharashtra, India. ${ }^{2}$ Department of Physiotherapy, Krishna Institute of \\ Medical Sciences (Deemed to Be University) Karad, Maharashtra, India.
}

\section{ABSTRACT}

\section{BACKGROUND}

The pre military trainee cadets are mostly unaware about proper training techniques and because of their lack of knowledge they are prone to have musculoskeletal problems especially their shoulder, spine, and knee joint are more prone to any injury. So, in order to avoid injuries, effort was made to make them aware about the risk of getting injured and proper fitness techniques which will prevent their injuries as well as help them to improve fitness level and field performance also. We wanted to evaluate the effect of advance strengthening program on physical fitness test performance in pre-military trainee cadets.

\section{METHODS}

In this comparative study, injury prone trainee cadets were randomly allocated in to two groups of 25 each with advanced training program. Group one did conventional type of routine exercise; group two followed advanced training protocol. Physical fitness test performance was assessed and compared between the two groups. Outcome measures were Burpees test, shoulder press, chin-ups, planks, leg press, 1600 meters running.

\section{RESULTS}

In Burpees test, $37 \%$ improvement was seen by conventional exercise program; whereas, it was $63 \%$ for advanced strengthening exercise program. In shoulder press, 38\% improvement was seen for conventional exercise program; whereas, it was $62 \%$ for advanced strengthening exercise program. In chin ups, $34 \%$ improvement was seen for conventional exercise program; whereas, it was $66 \%$ for advanced strengthening exercise program. In planks, 45\% improvement was seen with conventional exercise program; whereas, it was $55 \%$ for advanced strengthening exercise program. In leg press, 39\% improvement was seen with conventional exercise program; whereas, it was $61 \%$ with advanced strengthening exercise program. In 1600 meters running, 37\% improvement was seen with conventional exercise program; whereas, it was $63 \%$ by advanced strengthening exercise program. P value was $<0.0001$ which is significant.

\section{CONCLUSIONS}

A significant improvement was found in muscle performance, and occurrence of injury was seen to be reduced with advanced training program in injury prone trainee cadets.

\section{KEY WORDS}

Trainee Cadets, Injury, Chin-Ups, Burpees, Running, Shoulder Press, Leg Press, Planks
Corresponding Author: Dr. Sandeep Babasaheb Shinde, Associate Professor,

Faculty of Physiotherapy, Krishna Institute of Medical Sciences, Deemed to be University, Karad, Maharashtra, India. E-mail: drsandeepshinde24@gmail.com

DOI: $10.14260 /$ jemds/2020/424

How to Cite This Article:

Khutale M, Shinde SB. Effect of advanced strengthening program on physical fitness test performance in injury prone premilitary trainee cadets. J. Evolution Med. Dent. Sci. 2020;9(27):1948-1951, DOI: 10.14260/jemds/2020/424

Submission 01-03-2020,

Peer Review 04-06-2020,

Acceptance 10-06-2020,

Published 06-07-2020.

Copyright (C) 2020 JEMDS. This is an open access article distributed under Creative Commons Attribution License [Attribution 4.0 International (CC BY 4.0)] 


\section{BACKGROUND}

Physical fitness has been shown to be a difficult element in among new recruits who are taking army training. Individuals having a low level of physical fitness in army training have been shown to be more prone to injury and more likely to be unsuccessful for military recruitment than their fit counterparts. ${ }^{1}$ Musculoskeletal injuries especially those to the lower extremities are quite common in the military environment. ${ }^{2}$ Spinal problems and upper limb problems are second and third commonest problems observed in pre-military trainee cadets. Several risk factors has been recognized as associated to the high incidence of musculoskeletal injuries. These include adaptable risk factors such as volume of vigorous physical training, smoking, flexibility, footwear, training surfaces and training techniques as well as non-modifiable risk factors such as gender, age, anatomical variations, previous injury and past level of physical activity. ${ }^{3}$

Soft tissue/musculoskeletal injuries attracted by recruits undergoing basic army training continue to curse military organization attempting to train their entry level personnel. ${ }^{4}$ Injuries can cause pain and discomfort, lost training time, disruption of training schedule, increased health care expenditure and provider workload, permanent disability, extensive rehabilitation time and attrition of recruits unable to complete training. ${ }^{5}$ Incidence rates for injuries suffered by specific population including military recruits and athletes involved in specific sports activities has been recorded in several studies. ${ }^{6}$

Injuries significantly influence the department of army, approximately $25 \%$ of males and $50 \%$ of female recruits comfort one or more injuries during basic army training. ${ }^{7}$ These injuries consistently describe more than $80 \%$ of disability related medical discharge among first year recruits. ${ }^{8}$ Preventive strategies should be conducted at the primary factors subsidizing the risk factors for musculoskeletal injuries such as the amount and level of intensity of the training, level of physical fitness and probably equipment's (footwear). ${ }^{9}$ Specifically designed exercise program is very important to address all possible needs related to muscle performance and injury prevention. ${ }^{10}$ The specific approach to achieving higher levels of physical fitness that minimize injury rates in military trainees has limited access, so this study efforts are made to focus this highly significant problem mainly related to rural youth. This is targeted mainly to improve the physical fitness of the premilitary trainee cadets. ${ }^{11}$ After basic training, individuals need to maintain at least a moderate level of physical fitness not relatable to military job requirements. ${ }^{12}$

The pre military trainee cadets mostly don't have enough knowledge about proper training techniques and because of their lack of knowledge they mostly have musculoskeletal related problems and mostly there knee, ankle and shoulder joint get prone to any injury that's why to avoid injuries a small effort is made to give proper knowledge and proper fitness techniques which will prevent their injuries but as well help them to improve fitness level and field performance also. There is lack literature available on the Effect of advance strengthening program on physical fitness test performance in pre-military trainee cadets.
In India, most of the young population enter into the army training academy because of large number of unemployment and in such academies, they do not undergo training with proper techniques and guidance, as the trainees themselves have lack of patho-biomechanical and anatomical knowledge. So, they are at high risk of getting injured.

Because of getting injured, they are not eligible to get recruited in army. Due to this, their career gets destroyed and they get addicted to drugs. Because of unemployment, they enter into criminal world. They get mentally as well as physically unstable. There is literature available on military basic training candidates with injuries but not available on pre military trainee candidates who's having specific problems related with pre military trainee cadet's injury so there is need to study the effect of advance strengthening program on physical fitness test performance in pre-military trainee cadets.

\section{METHODS}

This comparative study was done to find the effect of advanced strengthening on physical fitness test performance in injury prone pre- military trainee cadets. The study was carried out in Karad. An approval of the study was obtained from the institutional ethical committee of Krishna Institute of Medical Sciences Deemed University. The purpose of the study was explained to the participants and consent form was taken. 50 pre- military trainee cadets were taken from Karad population for the study. Previous studies by JiménezOlmedo, J.M.; Penichet-Tomás, A.13 shown 21 male athletes. Minimum subjects required with $95 \%$ confidence and $80 \%$ power is $n=21$, since follow up study, $n=21+3$ (since $10 \%$ attrition rate) $=23$ Hence minimum 23 subjects in either group should be studied. In this interventional study injury prone trainee cadets were randomly allocated by using random allocation software in two groups of 25 each with advanced training program. The inclusion criteria were male pre- military trainee candidates of age group in between 18 to 27 years and fresh military cadets. The outcome measures taken to examine the improvement in pre- military trainee cadets after giving advanced strengthening protocol were taken as Burpees Test, Shoulder press, Chin- ups Planks, Leg press, 1600 meters respectively.

Group 1 was instructed to follow their routine conventional exercise program. (for example, running)

Group 2 the exercise protocol was designed. Which started with warm up session with minimum 10 minutes followed by sustained squats for 25 times. They were instructed to do side jumping 25 times and to repeat them in 3 sets, each set includes 25 side jumps between intervals of each set followed by $100 \mathrm{~m}$ sprint with resistance (using weight cuff tide at ankle). We also focused on upper body strengthening to improve their running posture and avoid further injuries caused because of bad running posture that also improved their running performance. After lower body training, we started with upper body training which included push ups (30) and bent knee pull ups (horizontal) (20) which they had to do twice a day with resisted shoulder shrugs (30) once a day. After completing training session, we asked them to cool down followed by prolonged stretching with icing. 
Statistical analysis and interpretation were done for each candidate to find out the effect of advanced strengthening on physical fitness test performance in injury prone pre- military trainee cadets.

\section{Injury Prevention Plan}

- Follow proper ergonomics.

- Use appropriate footwear while running.

- Avoid vigorous physical activity.

- Follow proper diet plan.

- Follow correct running pattern.

- Avoid running on irregular surface.

\section{Statistical Analysis}

The software Statistical Package Social Sciences (SPSS) v. 19.0 was used for the statistical analysis; Statistical Analysis was done by using unpaired t-Test.

\section{RESULTS}

\begin{tabular}{|cccc|}
\hline $\begin{array}{c}\text { Sr. } \\
\text { No. }\end{array}$ & $\begin{array}{c}\text { Demographic } \\
\text { Variables }\end{array}$ & $\begin{array}{c}\text { Number of } \\
\text { Participants }\end{array}$ & Percentage \\
1. & Age & 4 & \\
& $18-20$ & 36 & $10 \%$ \\
$21-22$ & & $90 \%$ \\
2 & Civilization & 29 & $72.50 \%$ \\
& Urban & 11 & $27.50 \%$ \\
3 & Rural & 15 & $37.50 \%$ \\
& Diet & 25 & $63 \%$ \\
\hline \multicolumn{4}{c}{} \\
& Veg & & \\
& Mixed & Table 1. Demographic Variables \\
\end{tabular}

\begin{tabular}{|ccccc|}
\hline Tests & Mean & $\begin{array}{c}\text { Standard } \\
\text { Deviation }\end{array}$ & $\begin{array}{c}\text { P } \\
\text { Value }\end{array}$ & $\begin{array}{c}\text { t } \\
\text { Value }\end{array}$ \\
Burpees Test (G1) & 16.48 & 3.00 & $<0.0001$ & 12.05 \\
Burpees Test (G2) & 27.56 & 5.34 & & \\
Shoulder Press (G1) & 23.6 & 5.05 & $<0.0001$ & 68.65 \\
Shoulder Press (G2) & 38.16 & 2.77 & & \\
Chin Ups (G1) & 4.52 & 1.08 & $<0.0001$ & 9.21 \\
Chin Ups (G2) & 8.64 & 1.95 & & \\
Planks (G1) & 34.72 & 5.16 & $<0.0001$ & 33.78 \\
Planks (G2) & 42.12 & 5.29 & & \\
Leg Press (G1) & 16.08 & 3.81 & $<0.0001$ & 31.23 \\
Leg Press (G2) & 24.88 & 3.98 & & \\
1600 m running (G1) & 5.61 & 0.94 & $<0.0001$ & 38.73 \\
1600 m running (G2) & 7.37 & 0.56 & \\
\hline \multicolumn{5}{|c|}{ Table 2. Post Exercise Results in Both Groups } \\
\hline \multicolumn{5}{|c}{}
\end{tabular}

Participants mean age was 21.92 years. After analysing and comparing the data significant effect was found on premilitary trainee cadets by advanced strengthening exercise program with improvement in physical fitness performance. Exercise protocol was given for 8 weeks and before and after physical fitness performance was assessed. The results which showed significant change are as follows:

In burpees test, $37 \%$ of improvement was seen by conventional exercise program whereas $63 \%$ by advanced strengthening exercise program. In shoulder press, $38 \%$ of improvement was seen by conventional exercise program whereas $62 \%$ by advanced strengthening exercise program. In chin ups, $34 \%$ of improvement was seen by conventional exercise program whereas $66 \%$ by advanced strengthening exercise program. In planks, $45 \%$ of improvement was seen by conventional exercise program whereas $55 \%$ by advanced strengthening exercise program. In leg press, 39\% of improvement was seen by conventional exercise program whereas $61 \%$ by advanced strengthening exercise program. In 1600 meters running, 37\% of improvement was seen by conventional exercise program whereas $63 \%$ by advanced strengthening exercise program. $\mathrm{P}$ value $<0.001$ was extremely significant.

\section{DISCUSSION}

The study is about the effect of advanced strengthening on physical fitness test performance in injury prone pre- military trainee cadets. Physical fitness is a remark of health and wellbeing and, more specifically, the skill to perform aspects of sports, occupations and daily activities. Physical fitness is usually achieved through proper nutrition, moderate vigorous physical exercise and enough rest. So, it is important to build our physical fitness to improve health and quality of life. The objective of this study was to find the effect of advanced strengthening on physical fitness test performance in injury prone pre- military trainee cadets and compare it with the conventional exercise program. Study was carried on 50 pre- military trainee cadets who were divide in two groups (25 each) and over a period of 8 weeks advanced strengthening exercise program was given and recorded and compared with the conventional exercise program. Group 1 was instructed to follow their routine conventional exercise program (for example running, push ups etc). Group 2 the exercise protocol was designed which started with warm up for minimum 10 minutes followed by sustained squats for 25 times. They were instructed to do side jumping 25 times and to repeat them in 3 sets, each set includes 25 side jumps between intervals of each set followed by $100 \mathrm{~m}$ sprint with resistance (using weight cuff tide at ankle). We also focused on upper body strengthening to improve their running posture and avoid further injuries caused because of bad running posture that also improved their running performance.

After lower body training, we started with upper body training which included push ups (30) and bent knee pull ups (horizontal) (20) which they had to do twice a day with resisted shoulder shrugs (30) once a day. After completing training session, we asked them to cool down followed by prolonged stretching with icing. Physical fitness plays an important role in maintaining optimum weight and not prone to any health problems. It is also important in maintaining relaxed state of mind. Significant change was found in between group 1 and group 2. In burpees test, 37\% of improvement was seen by conventional exercise program whereas $63 \%$ by advanced strengthening exercise program. In shoulder press, $38 \%$ of improvement was seen by conventional exercise program whereas $62 \%$ by advanced strengthening exercise program. In chin ups, $34 \%$ of improvement was seen by conventional exercise program whereas $66 \%$ by advanced strengthening exercise program. In planks, $45 \%$ of improvement was seen by conventional exercise program whereas $55 \%$ by advanced strengthening exercise program. In leg press, 39\% of improvement was seen by conventional exercise program whereas $61 \%$ by advanced strengthening exercise program. In 1600 meters running, $37 \%$ of improvement was seen by conventional exercise program whereas $63 \%$ by advanced strengthening 
exercise program. P value $<0.0001$ which indicates extremely significant. Physiotherapists can plan injury prevention protocol for pre-military trainee cadets. Hydrotherapy and aquatic exercises are beneficial for this purpose. ${ }^{14}$ The importance of physical training bridging course was highlighted for basic military training by Paola S. Wood et al. ${ }^{15}$ McKenzie and Lydia Anne recommended stress psycho-education and coping strategies be introduced early in the training phase for all trainees to improve overall training outcome. ${ }^{16}$

This a study was carried to find the effect of advanced strengthening on physical fitness test performance in injury prone pre- military trainee cadets. Fifty pre- military trainee cadets were included and divided into two groups of twentyfive each. Group one followed their conventional regular exercises and group two advanced strengthening exercise program was designed and was followed for a period of 8 weeks. Extremely significant results were found with $p$ value $<0.0001$. Physiotherapists play an important role and can design various protocols to get rid of this problem. From carrier point of view the pre-military training period is very important and young boys who are trying for job in defence and police recruitments are at risk of getting injury and may become disqualified for job. Salvatore Lo Bue et al pointed out in their study a strong relationship between hardiness and soldiers' physical performance. ${ }^{17}$ Team efforts are required to overcome these problems.

\section{CONCLUSIONS}

The study was carried to evaluate the effect of advanced strengthening on physical fitness test performance in injury prone pre-military trainee cadets. Data was assessed, and a significant decline was found in physical fitness performance with a $P$ value of $<0.0001$ which was extremely significant.

This study was funded by Krishna Institute of Medical Sciences Deemed to Be University Karad, Maharashtra.

\section{REFERENCES}

[1] Jones BH, Knapik JJ. Physical training and exerciserelated injuries: surveillance, research and injury prevention in military populations. Sports Med 1999;27(2):111-25.

[2] Almeida SA, Williams KM, Shaffer RA, et al. Epidemiological patterns of musculoskeletal injuries and physical training. Med Sci Sports Exerc 1999;31(8):1176-82.

[3] Cowan DN, Jones BH, Frykman PN, et al. Lower limb morphology and risk of overuse injury among male infantry trainess. Med Sci Sports Exerc 1996;28(8):94552.

[4] Kowal DM. Nature and causes of injuries in women resulting from an endurance training program. Am J Sports Med 1980;8(4):265-9.

[5] Gordon NF, Hugo EP, Cilliers JF. The South African Defence Force Physical Training Programme. Part III. Exertion- related injuries sustained at an SADF basic training centre. S Afr Med J 1986;69(8):491-4.

[6] Jones BH. Overuse injuries of the lower extremities associated with marching, jogging, and running: a review. Mil Med 1983;148(10):783-7.

[7] Technical Bulletin Medical 592. U.S. Department of the Army, Prevention and control of musculoskeletal injuries associated with physical training. Washington, DC, 2011. http://armypubs.army.mil/med/dr_a/pdf/tbmed592.pd $\mathrm{f}$

[8] Accession medical standards analysis \& research activity. Annual Report 2010 http://www.amasra.amedd.army.mil/reports/2010/AM SARA_Annual Report_2010.pdf

[9] Tomlinson JP, Lednar WM, Jackson JD. Risk of injury in soldiers. Mil Med 1987;152(2):60-4.

[10] Shinde SB, Varadharajulu G. Effect of therapeutic exercise programme in adults with early rheumatoid arthritis. Indian Journal of Physiotherapy and Occupational Therapy- An International Journal 2017;11(3):76-80.

[11] Knapik J, Ang P, Reynolds K, et al. Physical fitness, age, and incidence in infantry soldiers. J Occup Med 1993,35(6):598-603.

[12] Reinkar KA, Ozburne S. A comparison of male and female orthopaedic pathology in basic training. Mil Med 1979;144(8):532-6.

[13] Jiménez-Olmedo JM, Penichet-Tomás A, Pueo B, et al. Pattern of injuries in beach volleyball at the spanish national university championship. Revista Internacional de Medicina y Ciencias de la Actividad Física y del Deporte 2018;18(70):331-40.

[14] Sawant RS, Shinde SB. Effect of hydrotherapy based exercises for chronic nonspecific low back pain. Indian Journal of Physiotherapy \& Occupational Therapy 2019;13(1):149-54.

[15] Wood PS, Grant CC, du Toit PJ, et al. Effect of mixed basic military training on the physical fitness of male and female soldiers. Mil Med 2017;182(7):e1771-9.

[16] McKenzie LA. The psychological response of trainees during a 16 week regional policy academy: a needs assessment. The University of Arizona 2018.

[17] Lo Bue S, Kintaert S, Taverniers J, et al. Hardiness differentiates military trainees on behavioural persistence and physical performance. International Journal of Sport and Exercise Psychology 2018;16(4):354-64. 